\title{
Human papillomavirus like particles vaccine efficiently produced in a non-fermentative system based on insect larva
}

Alicia Fernández-San Millán ${ }^{1}$, Silvia Gómez-Sebastián², María C. Nuñez ${ }^{2}$, Jon Veramendi ${ }^{1}$ and José M. Escribano ${ }^{3 *}$

${ }^{1}$ Instituto de Agrobiotecnología (Universidad Pública de Navarra-CSICGobierno de Navarra), Campus Arrosadía, 31006 Pamplona, Spain

${ }^{2}$ Alternative Gene Expression S.L. (ALGENEX). Centro empresarial Parque Científico y Tecnológico de la Universidad Politécnica de Madrid Campus de Montegancedo, 28223 Pozuelo de Alarcón - Madrid, Spain

${ }^{3}$ Departamento de Biotecnología. INIA, Autovía A6 Km 7. 28040 Madrid, Spain

A. Fernández-San Millán and S. Gómez-Sebastián contributed equally to the paper

${ }^{*}$ Corresponding author 


\section{Summary}

Sexually transmitted human papillomavirus (HPV) infections cause most cervical cancers. The inability to propagate HPV in vitro has led to the development of alternative approaches to antigen production for vaccine development. In the present work we describe a low-cost efficient production of HPV virus-like particles (VLPs) in a non-fermentative system based on baculovirus and Trichoplusia $n i(T . n i)$ insects. The L1 protein from HPV 16 was expressed by a recombinant baculovirus in larva at levels that reached 18 to 21 $\mathrm{mg}$ per $\mathrm{g}$ of fresh insect biomass. It represents about 2.5 times the L1 production yields obtained per $\mathrm{g}$ of insect cells using the same recombinant baculovirus. Under electron microscopy, purified VLPs produced in larvae were indistinguishable from those produced in Sf-21 insect cell cultures, presenting identical shape and size. Immunization of mice with the insect-derived VLPs induced a potent immune response similar to that obtained with insect cellsderived VLPs. The use of live insect larvae as "mini bioreactors" opens up the possibility of cost-effective production of a vaccine against cervical cancer produced by papillomavirus, with special application in human populations where production costs restrain their use. 


\section{Introduction}

Human papillomavirus (HPV) is recognized as the main causative agent of invasive carcinomas of the uterine cervix (Sigurdsson et al., 2007). Cervical cancer is the most common cancer affecting women in developing countries, where $80 \%$ of deaths caused by this type of cancer occur. Among other considerations, affordability and cost-effectiveness of vaccination are the most important factors for application of HPV vaccines in developing countries. Two HPV types, HPV16 and HPV18, account for $60-70 \%$ of all cervical cancer cases worldwide (Ho et al., 1998; Munoz et al., 2003). The inability to propagate HPV in vitro has led to the development of alternative approaches to antigen production for vaccine development (Carter et al., 1991; Rose et al., 1993; Xi and Banks, 1991). Successful development of papillomavirus vaccines is dependent upon the use of nondenatured, conformationally correct capsid antigens forming pentamers or VLPs (Bonnez, Rose, and Reichman, 1992; Christensen and Kreider, 1990; Christensen et al., 1990; Christensen et al., 1992).

The viral capsid is primarily composed of 72 pentamers (capsomers) of the major late L1 protein, in association with 12 or more copies of the L2 protein. The L1 pentamers have the intrinsic property of self-assembly into empty capsids, referred to as virus like particles (VLPs) (Hagensee, Yaegashi, and Galloway, 1993; Kirnbauer et al., 1992; Kirnbauer et al., 1993; Rose et al., 1994; Zhou et al., 1993). VLPs are structurally and immunologically similar to infectious viruses, as determined by electron microscopy and their ability to bind conformation-dependent monoclonal antibodies (mAbs) (Kirnbauer et al., 1992). Conformational epitopes have been identified on the surface of HPV L1 VLPs (Christensen and Kreider, 1990). It is now well established that those epitopes are responsible for the activity of neutralizing antibodies (Christensen et al., 1994; White et al., 1998; White et al., 1999) and it has been demonstrated that high levels of neutralizing antibodies can be generated after immunization with HPV VLPs (Harro et al., 2001). 
Vaccines based on L1 capsid protein VLPs from several high risk HPV types have been proved to be effective against HPV infections. Raising high levels of neutralizing antibodies against each HPV type is believed to be the primary mechanism of protection, gained by vaccination. On June 8,2006 , the US FDA approved Gardasil, a prophylactic HPV vaccine from Merck. The vaccine showed protection against initial infection with the most common cancer inducers papillomaviruses, HPV types 16, 18, 6 and 11. GlaxoSmithKline obtained in 2007 the approval for another prophylactic vaccine targeting HPV types 16 and 18, known as Cervarix. Those vaccines are made up with recombinant protein $L 1$, expressed in yeast or in insect cells by recombinant baculoviruses infection (Gardasil and Cervarix respectively), self-assembled into VLPs similar to the wild-type virus. The use of these production systems based on fermentation technologies are expensive which is one of the reasons that justify the elevated price of the vaccines in the market, (a complete round of vaccination costs around $450 \$$ ).

In recent years, biotechnology has provided for the tools that allow the use of living organisms to produce recombinant vaccines with high efficiency but at a very low production costs. One of these alternatives is the use of insects as bioreactors (Liu, DeCarolis, and Beek, 2007). Recently, It has been described the obtention of rotavirus VLPs from infected Spodoptera frugiperda larvae using baculovirus, opening the option to get vaccines against rotavirus from insect larvae (Molinari, Peralta, and Taboga, 2008). There are other examples of recombinant subunit vaccines against infectious agents obtained from insects used as bioreactors (Chimeno Zoth et al., 2009; Hu, Yao, and Wu, 2008; Johansson, Price, and Kilbourne, 1995; Matsuoka et al., 1996; Pang et al., 2002). We currently have in clinical studies an insect larvae-derived recombinant subunit vaccine against RHDV (Perez-Filgueira et al., 2007).

Our main goal in this work was to exploit all possibilities of insects as biofactories to produce an effective HPV vaccine, always thinking in a dramatic reduction of production costs. In the present study we describe the expression of the L1 protein from HPV type 16 in Trichoplusia ni larvae. L1 protein self- 
assembled forming VLPs in the larvae tissues and the immune response of larva-derived VLPs compared to that induced by VLPs obtained in insect cell cultures in immunized mice was similar.

\section{Experimental procedures}

\section{Construction of the Baculovirus expression vector}

HPV-16 L1 cDNA (GenBank Accession AF472508) was provided by Dr. Robert D. Burk (Albert Einstein College of Medicine, New York, USA). For cloning into the pFastBac ${ }^{\top \mathrm{M}} 1$ vector (Invitrogen), $L 1$ was amplified by PCR with the following primers in order to introduce the Afl III and Not I sites: 5'CCACATGTCTCTTTGGCTGCCTAGCG-3' and 5'-GCGGCCGCTCGAGTTA CAGCTTACGTTTTTTGC-3'. The $L 1$ gene was cloned into the pGEM-T vector (Promega), digested with Not I, treated with alkaline phosphatase and introduced into the pFastBac ${ }^{\mathrm{TM}} 1$ vector of the Bac-to-Bac Baculovirus Expression System (Invitrogen). The final clone was sequenced, and used to transfer $\mathrm{DH} 10 \mathrm{Bac}^{\mathrm{TM}} E$. coli cells for the transposition into the recombinant bacmid. A minimum of $1 \mu \mathrm{g}$ of this bacmid was used to transfect $1 \times 10^{6}$ Spodoptera frugiperda Sf-21 cells using Cellfectin Reagent (Invitrogen). The P1 viral stock was obtained after $72 \mathrm{~h}$ of incubation at $28^{\circ} \mathrm{C}$. The baculovirus was amplified following the manufacturer's instructions.

\section{Larvae and insect cell infection}

Trichoplusia ni ( $T$. ni, Cabbage looper) larvae were reared under level-2 biosafety conditions following previously described methodology (PerezFilgueira et al., 2006). For all experiments, fifth-instar larvae were injected with the recombinant baculoviruses near the proleg (forward the body cavity) using $10 \mu \mathrm{l}$ of different pfu/larva doses, as indicated on each experiment. At different postinfection times, larvae were collected, immediately frozen and kept at $-20^{\circ} \mathrm{C}$ until processed. 
Preconfluent monolayers of Sf-21 insect cells were infected with 10 pfu (plaque forming units) of the recombinant baculoviruses and harvested $72 \mathrm{hpi}$ (hours post-infection).

\section{Western blot and Coomassie staining analysis}

T. ni larvae were homogenized in $10 \mathrm{vol}$ of phosphate buffered saline $0.5 \mathrm{M}$ $\mathrm{NaCl}, \mathrm{pH} 7.4$ (PBS-HS) and sonicated for 20 seconds. After centrifugation at $20000 \mathrm{~g}$ for $5 \mathrm{~min}$, the supernatant was considered the soluble fraction. Sf-21 cells were disrupted with RIPA extraction buffer $(150 \mathrm{mM} \mathrm{NaCl}, 1 \% \mathrm{NP}-40$, $0.1 \%$ SDS, $50 \mathrm{mM}$ Tris $\mathrm{pH} 8,5 \mathrm{mM} \beta$-mercaptoethanol and a protein inhibitor cocktail (Complete, Roche, Germany), kept on ice for $30 \mathrm{~min}$, centrifuged at $5000 \mathrm{rpm}$ for $5 \mathrm{~min}$, and the supernatant was collected for further assays.

Proteins were separated on $10 \%$ SDS (w/v) polyacrylamide gels (SDS-PAGE) and were stained with Coomassie Brilliant Blue G-250 (BioRad) or transferred onto nitrocellulose membranes (Hybond C, GE Healthcare). The membranes were blocked overnight in PBS with $0.1 \%(\mathrm{v} / \mathrm{v})$ Tween 20 (PBS-T) and 4\% skimmed milk (PBS-TM), and incubated for $1 \mathrm{~h}$ with a primary antibody, Cam Vir-1 (Abcam) diluted 1:25000 in PBS-TM. After 2 washes of 10 min in PBS-T, membranes were incubated for $1 \mathrm{~h}$ with a peroxidase-conjugated rabbit antimouse IgG (Sigma) diluted 1:25000 in PBS-TM. After washing, the specific signal was detected using the Advanced ECL system (GE Healthcare) according to the manufacturer's instructions.

\section{ELISA quantification of HPV-16 L1 protein}

Infected or uninfected larvae or insect cells soluble protein extracts were pelleted at $1500 \mathrm{rpm}$. Samples were resuspended in $10 \mathrm{vol}(\mathrm{w} / \mathrm{v})$ of PBS-HS, sonicated for $10 \mathrm{~s}$, and centrifuged at $20000 \mathrm{~g}$ for $5 \mathrm{~min}$ at $4^{\circ} \mathrm{C}$ to remove cellular debris. Samples were incubated in a 96 well polyvinyl chloride microtiter plate (Costar, Corning) overnight at $4^{\circ} \mathrm{C}$. Wells were blocked with PBS-TM $1 \%$ skimmed milk for $1 \mathrm{~h}$ at RT, washed three times with PBS-T and incubated with anti-L1 H16.V5 mAb (provided by Neil Christensen, Pennsylvania State University) at 1:500 in PBS-TM $\left(1 \mathrm{~h}\right.$ at $\left.37^{\circ} \mathrm{C}\right)$. Wells were washed three times with PBS-T and incubated with a 1:1500 dilution of a rabbit anti-mouse IgG- 
peroxidase conjugate in PBS-TM $\left(1 \mathrm{~h}\right.$ at $\left.37^{\circ} \mathrm{C}\right)$. After three washes with PBS-T, plates were developed with ABTS [2, 2'-azino-bis (3-ethylbenzthiazoline-6sulfonic) acid] (Roche). The reaction was read at $405 \mathrm{~nm}$ in a microtiter plate reader (Multiskan Ex, Labsystems). The standard curve to calculate the amount of recombinant protein with H16.V5 mAb was made by plating purified VLPs produced in insect cells by baculovirus infection, as described below. VLPs were used in the range of 30-150 $\mathrm{ng}$ per well diluted in $100 \mathrm{mM}$ PBS pH 7.4 buffer. Larvae extracts containing L1 protein were diluted (1:1000 to 1:8000) to fit within the linear range of the standards

\section{Purification of VLPs}

Insect cells

For the production of VLPs, Sf-21 insect cells were grown to a density of 1-1.2 $\mathrm{x}$ $10^{6}$ cells $/ \mathrm{mL}$ on BD BaculoGold ${ }^{\mathrm{TM}}$ TNM-FH Insect Medium (BD Biosciences) supplemented with $50 \mu \mathrm{g} / \mathrm{mL}$ gentamycin, 50 units $/ \mathrm{mLe}$ penicillin and $50 \mu \mathrm{g} / \mathrm{mL}$ streptomycin in $75 \mathrm{~cm}^{2}$ flasks. Cells were then infected at a m.o.i (multiplicity of infection) of 1-5 and 72 hpi (hours post-infection) cells were pelleted by centrifugation at $1000 \mathrm{~g}$ for $5 \mathrm{~min}$.

$500 \mathrm{mg}$ of pelleted infected insect cells were resuspended in $8 \mathrm{~mL}$ PBS-HS and sonicated for $2 \mathrm{~min}$. Extracts were layered onto a $40 \%$ sucrose cushion and centrifuged in a swinging bucket rotor (Kontron TST4114) for $2 \mathrm{~h}$ at $140000 \mathrm{~g}$ at $4^{\circ} \mathrm{C}$. The resulting pellets were resuspended in $10 \mathrm{~mL}$ of a $27 \%(\mathrm{w} / \mathrm{v}) \mathrm{CsCl}$ in PBS-HS solution and centrifuged for $20 \mathrm{~h}$ at $260,000 \mathrm{~g}$ in a swinging bucket rotor at $10^{\circ} \mathrm{C}$. $500 \mu \mathrm{L}$ fractions were collected and their densities were measured with a refractometer. The desired fractions were dialyzed overnight against PBS-HS.

\section{Larvae}

For extraction of larvae VLPs, $500 \mathrm{mg}$ of fresh weight larvae were homogenized in a blender with $8 \mathrm{~mL}$ of PBS-HS and sonicated for $2 \mathrm{~min}$. After centrifugation at $20000 \mathrm{~g}$ for $5 \mathrm{~min}$ at $4^{\circ} \mathrm{C}$, supernatants were layered onto a $40 \%$ sucrose 
cushion. The rest of the protocol was similar to that used for insect cell VLP extraction.

\section{Electron microscopy and immunogold labelling}

For negative staining, $20 \mu \mathrm{L}$ of samples of the positive fractions in the $\mathrm{CsCl}$ gradient were dialyzed against PBS-HS on floating filter pads (0.2 $\mu \mathrm{m}$ pore size, Millipore). Samples were placed onto carbon-coated copper grids (400 mesh size) covered with Formvar membrane and stained with $1 \%$ uranyl acetate solution for $1 \mathrm{~min}$. Samples were inspected under a ZEISS EM 910 transmission electron microscope (TEM) operating at 60 and $80 \mathrm{kV}$.

\section{Analysis of L1 assembly by sucrose sedimentation}

To identify different assembly forms of $L 1$, soluble extracts from insect cells and larvae (200 mg/ 2mL PBS-HS) were prepared as previously described for western blotting. Samples were loaded onto linear 10 to $65 \%$ (w/v) sucrose gradients. After $2 \mathrm{~h}$ centrifugation at $150000 \mathrm{~g}$ in a swinging bucket rotor, 20 fractions of $500 \mu \mathrm{L}$ were collected, determined their density by refractometry and analysed by ELISA with the Cam Vir-1 (Abcam) antibody. Sedimentation of L1 capsomers was calibrated with catalase from bovine liver (Sigma) as sedimentation marker (11.3S).

\section{Immunization of animals}

Female Balb/c mice were purchased from Harlan Ibérica (Barcelona, Spain). Groups of 8 -week-old mice $(n=6)$ were immunized by intraperitoneal injection $(0.2 \mathrm{~mL})$ with $\mathrm{CsCl}$ purified VLPs from larvae (as described before) in presence of complete Freund's adjuvant. The amount of VLPs injected was $5 \mu \mathrm{g}$ per mouse. As a negative control, a group of mice $(n=6)$ was intraperitoneally immunized with larvae extracts from BacNi (baculovirus with no insert) infected larvae as negative control. As a positive control, a group of mice $(n=6)$ was intraperitoneally immunized with $5 \mu \mathrm{g}$ of VLPs from insect cell cultures purified by $\mathrm{CsCl}$. Animals were boosted at days 21 and 35 (using incomplete Freund's Adjuvant). Blood samples were collected from the facial vein at days 0, 21, 35 and 50. Pooled sera were titrated against purified VLPs produced in yeast (Gardasil, Merck) by ELISA in microtitre plates coated overnight at $4^{\circ} \mathrm{C}$ with 50 
$\mu L$ of yeast-derived VLPs (100 ng of L1-VLPs per well) diluted in PBS ( $\mathrm{pH} 7.4$ ). Subsequently, the wells were blocked $1 \mathrm{~h}$ at room temperature with PBS-TM and then incubated $1 \mathrm{~h}$ at $37^{\circ} \mathrm{C}$ with serial dilutions of pooled sera in PBS-TM. Then, plates were incubated for $1 \mathrm{~h}$ at $37^{\circ} \mathrm{C}$ with a goat anti-mouse IgG antibody horseradish peroxidase conjugate. Washes were performed between each step with PBS-T. Plates were developed by adding ABTS. Absorbance at $405 \mathrm{~nm}$ was measured in a microtiter plate reader. Antibody titres were expressed as the highest serum dilution to yield twice the absorbance mean of pre-immune sera. 


\section{Results}

\section{Expression of HPV-16 L1 in Sf-21 cells and insect larvae}

HPV-16 L1 ORF was cloned into the baculovirus transfer vector pFasBac giving the plasmid named $\mathrm{pL} 1$, which was used to obtain a recombinant baculovirus (BacL1) as described in Methods. To study the expression of the L1 protein in $T$. $n i$ insect larvae, SDS-PAGE of soluble and insoluble protein larvae fractions was made using as a control an insect cell His-tag L1 protein purified by affinity chromatography. Coomassie blue-stained gels showed that L1 was easily identified as a major band with mobility around $55 \mathrm{kDa}$ (Fig. 1a). Most L1 protein was detected in the soluble fraction with identical mobility to that observed with L1 protein expressed in insect cells. The specificity of the expressed protein was further characterized by western blot using the Cam Vir1 antibody (Fig. 1b).

Once the L1 expression was demonstrated, the optimal L1 expression conditions induced by BacL1 in larvae were analized.. Fifth instar larvae were inoculated with three different doses of BacL1 virus. Samples were processed at different times post-inoculation and analyzed by western blot. A $55 \mathrm{KDa}$ band corresponding to the $L 1$ protein was accumulated in inoculated larvae in a timedependent manner (Fig. 1C). A specific ELISA quantification also confirmed these observations (Fig. 1C), determining optimal conditions for L1 expression with an infection dose of $4 \times 10^{4}-10^{5}$ pfu/larva at $72 \mathrm{hpi}$. At later post-inoculation time the larvae productivity use to drop dramatically.

In order to determine the larvae expression efficiency we compared it with the insect cell commercial system and a recently described method of chloroplast transformation of tobacco for L1 vaccine production (Fernandez-San Millan et al., 2008) was carried out. As it is shown in figure 1d, the L1 recombinant protein reached accumulation levels ranging from 18 to $21 \mathrm{mg}$ per $\mathrm{g}$ of insect biomass (about four insect larva). Insect larvae produced about 2.5 and 5 times more L1 protein than insect cell cultures and transgenic chloroplasts respectively per biomass unit. Therefore, insect larvae are the most efficient expression system for $\mathrm{L} 1$ protein described to date. 


\section{Characterization of larvae produced virus-like particles.}

Since a successful development of a papilloma vaccine is dependent upon conformationally correct capsid antigens forming pentamers or VLPs, we investigated the L1 self-assembling in larvae tissues. For that purpose, total soluble protein fractions obtained from BacL1-infected larvae were used for HPV VLPs purification using $\mathrm{CsCl}$ and sucrose gradients. A clear antigenic peak measured by ELISA, using the Cam Vir-1 anti-L1 mAb as primary antibody, was detected in the fraction 4 , collected from the $\mathrm{CsCl}$ gradient both with material derived from insect cell cultures or insect larvae (Fig. 2a). L1 protein was mainly present in $\mathrm{CsCl}$ gradient fractions at a density of $1.27 \mathrm{~g} / \mathrm{mL}$, suggesting the presence of VLPs. In both systems the peaks of L1 protein derived from insect cells and larvae overlapped indicating that complex structures were very similar in densities (Fig. 2a). Fractions where the maximum L1 was found either in cells or in larvae extracts were also positive in immunoblot assay (Fig. 2b).

Analysis of fraction 4 of the $\mathrm{CsCl}$ gradient by electron microscopy using negative staining showed structures that correspond in shape and size to previously described HPV VLPs. They consisted of spherical particles of about $55 \mathrm{~nm}$ in diameter with a regular array of capsomeres. Smaller, larger, and irregular spheres as well as tubular structures were also seen (Fig. 2c). These structures have been already described by others in plants (Biemelt et al., 2003; Maclean et al., 2007) but also in insect cells (Le Cann et al., 1995; Volpers et al., 1994).

Soluble proteins from infected larvae and insect cell cultures were also submitted to sucrose sedimentation analysis in order to address L1 selfassembly patterns. Gradient fractions were collected and analyzed by ELISA with the Cam Vir-1 mAb. Results revealed that the L1 antigen assembled into similar structures independently of the production method used (insect cell cultures or larvae), presenting overlapping peaks of L1 distribution at specific 
sucrose densities measured by refractometry (Fig. 2d). Either there were not capsomers in fraction 17 nor monomers in fraction 20.

\section{Immunogenicity of purified HPV 16 VLPs produced in insect larva}

To investigate the immunogenicity of larvae-derived HPV VLPs, mice (5 Balb/c per group) were intraperitoneally immunized with $5 \mu \mathrm{g}$ of purified VLPs derived from larvae and the immune response was compared to that induced by similar preparations derived from Sf-21 insect cells. Animals were boosted at days 21 and 35 after the first vaccine dose. The L1-specific humoral responses in mice were analyzed by L1 VLPs-based ELISA using native yeast-derived VLPs as antigen to coat the plates (Fig. 3). At day 50 post inoculation, all immunized animals reached titres higher than 1/10000. In the case of the animals immunized with the purified VLPs from larvae the titres were even higher than those derived from mice immunized with insect cells derived VLPs (Fig. 3). All control mice immunized with extracts from infected larvae with a non-related baculovirus and equally processed did not show any specific immune response cross-reactive with L1 protein (Fig. 3). 


\section{Discussion}

HPV infection is a necessary factor in the development of nearly all cases of cervical cancer. Although the widespread use of Pap tests (Papanicolau test) has reduced the incidence and lethality of cervical cancer in developed countries, the disease still kills several hundred thousand women per year worldwide. More than any other cancer, cervical cancer reflects striking global health inequity. It is the second most common cancer among women worldwide, with about 493,000 new cases diagnosed annually. Of 274000 deaths due to cervical cancer each year, more than $80 \%$ occur in developing countries, and this proportion is expected to increase to $90 \%$ by 2020 (Agosti and Goldie, 2007). Affecting relatively young women, it is the largest single cause of years of life lost by cancer in the developing world. Considerations for policymakers debating the use of HPV vaccine in any particular country include among other factors cost-effectiveness of vaccination relative to other programs competing for resources. The factors with the greatest influence on the cost-effectiveness of vaccination will be the price of the vaccine and the costs of a program to reach adolescents. These make urgent to develop lower production costs HPV vaccines for developing countries. The main objective of this work was the search for an HPV vaccine production technology that facilitate a potential dramatic cost reduction in order to facilitate its application in developing countries or extend the vaccination to other susceptible population. For such purpose, we have explored in this work the use of a non-fermentative system based on insects ( $T$. ni larvae) as biofactories in combination with a recombinant baculovirus to produce HPV VLPs.

The major structural protein of the HPV capsid, L1, is the antigen of choice for the development of prophylactic vaccines. It is well known that expression of the L1 by recombinant baculoviruses in insect cell cultures is sufficient for the formation of VLPs which are highly immunogenic (Rose et al., 1993). Interestingly one of the two approved prophylactic HPV vaccines is expressed in insect cell cultures by fermentation technology by recombinant baculoviruses (Cervarix). However, although this technology platform is very efficient in 
recombinant protein production and generates highly immunogenic selfassembled L1 derived VLPs, the production costs increase the vaccine price at levels that can not be easily applied for HPV prevention programs in developing countries. To overcome this problem, other heterologous systems to produce L1 have been developed as bacteria, eukaryotic cell cultures or transgenic plants (Aires et al., 2006; Fernandez-San Millan et al., 2008; Fraillery et al., 2007; Heino, Dillner, and Schwartz, 1995; Kim et al., 2007; Maclean et al., 2007). However, none of them allow a significant cost reduction that would boost its wide use in developing countries.

It has been extensively demonstrated that the larvae expression system is a suitable method for inexpensive production of large amounts of recombinant proteins for either vaccine or diagnostic use (Barderas et al., 2000; Ferrer et al., 2007; Gomez-Sebastian et al., 2008; Lopez et al., 2005; Perez-Filgueira et al., 2006; Perez-Filgueira et al., 2007). In this work we have demonstrated the high yield production of L1 protein in $T$. ni larvae that self-assembles forming the same type of VLPs that are currently used in vaccine formulations against this important infectious disease. One gram of infected insect biomass is able to produce in optimal conditions $18-21 \mathrm{mg}$ of totally soluble recombinant L1 protein This means a 2.5 times the L1 production yields obtained in insect cell cultures and 5 times more productivity than transplastomic tobacco plants per biomass unit (Fernandez-San Millan et al., 2008). Another interesting feature of the insect platform technology is that the system requires lower amounts of baculovirus inocula than the insect cell cultures for protein expression optimization. This is of great interest from a practical point of view since the production cost of the baculovirus stocks could be also reduced. Furthermore, the necessity to generate high titre virus stocks and the difficulty to get them would be also avoided with the larvae expression system. Additionally, recombinant baculoviruses can be propagated in insect larvae without the need of insect cell cultures avoiding any possibility of the use of any animal compound (ie. animal sera) in the production process.

The reactivity of the larva-derived L1 structures with the conformational-specific anti-HPV antibody H16.V5 mAb, strongly suggest a correct conformation of the 
HPV VLPs generated in the larva system. Additionally, the data obtained from $\mathrm{CsCl}$ or sucrose sedimentation gradients related to sedimentation coefficient of L1 structures obtained from larva, as well as the electron microscopy observations, corroborates that VLPs formed are similar to that obtained by other vaccine production systems. As L1 protein expressed in insect cells, L1 obtained by infected larvae was present in $\mathrm{CsCl}$ gradient fractions at a density of $1.27 \mathrm{~g} / \mathrm{mL}$, in which it is known that HPV VLPs migrates. In addition, as observed by sucrose gradient sedimentation, L1 structural pattern found in larva overlaps with that obtained in insect cell cultures. Electron microscopy negative staining of sedimentation fractions revealed also the presence of indistinguisible VLPs to that produced in insect cell cultures (Fig. 2c).

Mice immunized with HPV VLPs derived from larva or insect cell cultures showed very good humoral immune responses, with antibody titers higher than 1/10000 after two vaccine boosts. Up to date, capsomers are considered to be a cost-effective alternative to VLP-based vaccines because they can be produced in bacterial expression systems and because of their high degree of stability. However, it has been already demonstrated that VLPs induced, independently of the route of immunization, significantly higher titers of neutralizing antibodies than capsomers (Fligge et al., 2001). The insect expression system is a very competitive alternative to bacterial expression systems in terms of production costs, with the advantage of the insect cells in terms of facilitate high complex structures formation such L1 VLPs instead only capsomers as those produced in bacteria systems.

For countries with a gross domestic product of less than $\$ 1000$ per capita, the per-dose cost may need to be as low as $\$ 1$ to $\$ 2$ to make vaccination both costeffective and affordable (Agosti and Goldie, 2007). Without a doubt, one of the greatest barriers to the introduction of this vaccine is price. Using the larvae expression system, the high production costs of current HPV vaccines would be dramatically reduced, allowing their distribution in developing countries, where the highest incidence of this disease occurs. It is important to remark that with every 5 -year delay in bringing vaccination to developing countries, 1.5 million to 2 million more women will die (Agosti and Goldie, 2007). 
Another concern is that by eliminating the two major oncogenic HPV genotypes through vaccination, current HPV vaccines target only two oncogenic types (albeit those responsible for $70 \%$ of cervical cancers), the remaining oncogenic types will become more common to fill a niche previously occupied by HPV16 and HPV18, thereby reducing the impact of the current vaccines on cervical cancer. However a low-cost multivalent HPV vaccine eliciting protection against a broad spectrum of HPV types would be particularly useful where national screening programs are currently not instituted. Our approach could be the clue to afford the challenge of a viable worldwide vaccination because its simplicity, easily testing and manufacture of such complex vaccines at a low cost. The scalability of vaccine production in the larva platform is immediate. In less than 5 weeks it is possible to generate a new recombinant baculovirus and initiate a massive production in larva without minor optimization procedures.

Acknowledgements

We thank to Robert D. Burk for providing HPV-16 L1 cDNA, Neil Christensen for providing H16.V5-specific mAb, Nuria Cortadellas for technical assistance in electron microscopy and Ignacio Segovia for technical assistance in mice inoculation. This work was partially supported by Grants BIO2005-00155 and CPE03-022-C5-2 from the Ministerio de Educación y Ciencia and INIA to Jon Veramendi and funds from Alternative Gene Expression S.L (ALGENEX). 


\section{References}

Agosti, J. M., and Goldie, S. J. (2007). Introducing HPV vaccine in developing countries--key challenges and issues. N Engl J Med 356(19), 1908-10.

Aires, K. A., Cianciarullo, A. M., Carneiro, S. M., Villa, L. L., Boccardo, E., Perez-Martinez, G., Perez-Arellano, I., Oliveira, M. L., and Ho, P. L. (2006). Production of human papillomavirus type $16 \mathrm{L1}$ virus-like particles by recombinant Lactobacillus casei cells. Appl Environ Microbiol 72(1), 745-52.

Barderas, M. G., Wigdorovitz, A., Merelo, F., Beitia, F., Alonso, C., Borca, M. V., and Escribano, J. M. (2000). Serodiagnosis of African swine fever using the recombinant protein p30 expressed in insect larvae. J Virol Methods 89(1-2), 129-36.

Biemelt, S., Sonnewald, U., Galmbacher, P., Willmitzer, L., and Muller, M. (2003). Production of human papillomavirus type 16 virus-like particles in transgenic plants. $J$ Virol 77(17), 9211-20.

Bonnez, W., Rose, R. C., and Reichman, R. C. (1992). Antibody-mediated neutralization of human papillomavirus type 11 (HPV-11) infection in the nude mouse: detection of HPV-11 mRNAs. J Infect Dis 165(2), 376-80.

Carter, J. J., Yaegashi, N., Jenison, S. A., and Galloway, D. A. (1991). Expression of human papillomavirus proteins in yeast Saccharomyces cerevisiae. Virology 182(2), 513-21.

Chimeno Zoth, S., Gomez, E., Carballeda, J. M., Taboga, O., Carrillo, E., and Berinstein, A. (2009). Potential use of the hemagglutinin-neuraminidase glycoprotein of Newcastle disease virus expressed in Rachiplusia nu larvae as an immunogen for chickens. Clin Vaccine Immunol 16(5), 775-8.

Christensen, N. D., Hopfl, R., DiAngelo, S. L., Cladel, N. M., Patrick, S. D., Welsh, P. A., Budgeon, L. R., Reed, C. A., and Kreider, J. W. (1994). Assembled baculovirusexpressed human papillomavirus type $11 \mathrm{~L} 1$ capsid protein virus-like particles are recognized by neutralizing monoclonal antibodies and induce high titres of neutralizing antibodies. J Gen Virol 75 ( Pt 9), 2271-6.

Christensen, N. D., and Kreider, J. W. (1990). Antibody-mediated neutralization in vivo of infectious papillomaviruses. J Virol 64(7), 3151-6.

Christensen, N. D., Kreider, J. W., Cladel, N. M., Patrick, S. D., and Welsh, P. A. (1990). Monoclonal antibody-mediated neutralization of infectious human papillomavirus type 11. J Virol 64(11), 5678-81.

Christensen, N. D., Kreider, J. W., Shah, K. V., and Rando, R. F. (1992). Detection of human serum antibodies that neutralize infectious human papillomavirus type 11 virions. $J$ Gen Virol 73 ( Pt 5), 1261-7.

Fernandez-San Millan, A., Ortigosa, S. M., Hervas-Stubbs, S., Corral-Martinez, P., SeguiSimarro, J. M., Gaetan, J., Coursaget, P., and Veramendi, J. (2008). Human papillomavirus L1 protein expressed in tobacco chloroplasts self-assembles into virus-like particles that are highly immunogenic. Plant Biotechnol J 6(5), 427-41.

Ferrer, F., Zoth, S. C., Calamante, G., and Taboga, O. (2007). Induction of virus-neutralizing antibodies by immunization with Rachiplusia nu per os infected with a recombinant baculovirus expressing the E2 glycoprotein of bovine viral diarrhea virus. I Virol Methods 146(1-2), 424-7.

Fligge, C., Giroglou, T., Streeck, R. E., and Sapp, M. (2001). Induction of type-specific neutralizing antibodies by capsomeres of human papillomavirus type 33. Virology 283(2), 353-7.

Fraillery, D., Baud, D., Pang, S. Y., Schiller, J., Bobst, M., Zosso, N., Ponci, F., and NardelliHaefliger, D. (2007). Salmonella enterica serovar Typhi Ty21a expressing human 
papillomavirus type $16 \mathrm{~L} 1$ as a potential live vaccine against cervical cancer and typhoid fever. Clin Vaccine Immunol 14(10), 1285-95.

Gomez-Sebastian, S., Perez-Filgueira, D. M., Gomez-Casado, E., Nunez, M. C., SanchezRamos, I., Tabares, E., and Escribano, J. M. (2008). DIVA diagnostic of Aujeszky's disease using an insect-derived virus glycoprotein E. J Virol Methods 153(1), 29-35.

Hagensee, M. E., Yaegashi, N., and Galloway, D. A. (1993). Self-assembly of human papillomavirus type 1 capsids by expression of the L1 protein alone or by coexpression of the L1 and L2 capsid proteins. J Virol 67(1), 315-22.

Harro, C. D., Pang, Y. Y., Roden, R. B., Hildesheim, A., Wang, Z., Reynolds, M. J., Mast, T. C., Robinson, R., Murphy, B. R., Karron, R. A., Dillner, J., Schiller, J. T., and Lowy, D. R. (2001). Safety and immunogenicity trial in adult volunteers of a human papillomavirus 16 L1 virus-like particle vaccine. J Natl Cancer Inst 93(4), 284-92.

Heino, P., Dillner, J., and Schwartz, S. (1995). Human papillomavirus type 16 capsid proteins produced from recombinant Semliki Forest virus assemble into virus-like particles. Virology 214(2), 349-59.

Ho, G. Y., Bierman, R., Beardsley, L., Chang, C. J., and Burk, R. D. (1998). Natural history of cervicovaginal papillomavirus infection in young women. N Engl J Med 338(7), 423-8.

$\mathrm{Hu}, \mathrm{Y}$. C., Yao, K., and Wu, T. Y. (2008). Baculovirus as an expression and/or delivery vehicle for vaccine antigens. Expert Rev Vaccines 7(3), 363-71.

Johansson, B. E., Price, P. M., and Kilbourne, E. D. (1995). Immunogenicity of influenza A virus N2 neuraminidase produced in insect larvae by baculovirus recombinants. Vaccine 13(9), 841-5.

Kim, S. N., Jeong, H. S., Park, S. N., and Kim, H. J. (2007). Purification and immunogenicity study of human papillomavirus type 16 L1 protein in Saccharomyces cerevisiae. $J$ Virol Methods 139(1), 24-30.

Kirnbauer, R., Booy, F., Cheng, N., Lowy, D. R., and Schiller, J. T. (1992). Papillomavirus L1 major capsid protein self-assembles into virus-like particles that are highly immunogenic. Proc Natl Acad Sci U S A 89(24), 12180-4.

Kirnbauer, R., Taub, J., Greenstone, H., Roden, R., Durst, M., Gissmann, L., Lowy, D. R., and Schiller, J. T. (1993). Efficient self-assembly of human papillomavirus type $16 \mathrm{L1}$ and L1-L2 into virus-like particles. J Virol 67(12), 6929-36.

Le Cann, P., Chabaud, M., Leboulleux, D., Mougin, C., Mayelo, V., Legrand, M. C., Calvet, C., Afoutou, J. M., Coll-Seck, A. M., and Coursaget, P. (1995). Detection of antibodies to L1, L2, and E4 proteins of human papillomavirus types 6,11 , and 16 by ELISA using synthetic peptides. J Med Virol 45(4), 410-4.

Liu, Y., DeCarolis, N., and Beek, N. (2007). Protein production with recombinant baculoviruses in lepidopteran larvae. Methods Mol Biol 388, 267-80.

Lopez, M. G., Peralta, A., Berinstein, A., Fondevila, N., Carrillo, E., and Taboga, O. (2005). High-level expression of recombinant $3 A B 1$ non-structural protein from FMDV in insect larvae. J Virol Methods 124(1-2), 221-4.

Maclean, J., Koekemoer, M., Olivier, A. J., Stewart, D., Hitzeroth, II, Rademacher, T., Fischer, R., Williamson, A. L., and Rybicki, E. P. (2007). Optimization of human papillomavirus type 16 (HPV-16) L1 expression in plants: comparison of the suitability of different HPV-16 L1 gene variants and different cell-compartment localization. J Gen Virol 88(Pt 5), 1460-9.

Matsuoka, H., Kobayashi, J., Barker, G. C., Miura, K., Chinzei, Y., Miyajima, S., Ishii, A., and Sinden, R. E. (1996). Induction of anti-malarial transmission blocking immunity with a recombinant ookinete surface antigen of Plasmodium berghei produced in silkworm larvae using the baculovirus expression vector system. Vaccine 14(2), 120-6. 
Molinari, P., Peralta, A., and Taboga, O. (2008). Production of rotavirus-like particles in Spodoptera frugiperda larvae. J Virol Methods 147(2), 364-7.

Munoz, N., Bosch, F. X., de Sanjose, S., Herrero, R., Castellsague, X., Shah, K. V., Snijders, P. J., and Meijer, C. J. (2003). Epidemiologic classification of human papillomavirus types associated with cervical cancer. $N$ Engl J Med 348(6), 518-27.

Pang, A. L., Hashimoto, C. N., Tam, L. Q., Meng, Z. Q., Hui, G. S., and Ho, W. K. (2002). In vivo expression and immunological studies of the 42-kilodalton carboxyl-terminal processing fragment of Plasmodium falciparum merozoite surface protein 1 in the baculovirus-silkworm system. Infect Immun 70(6), 2772-9.

Perez-Filgueira, D. M., Gonzalez-Camacho, F., Gallardo, C., Resino-Talavan, P., Blanco, E., Gomez-Casado, E., Alonso, C., and Escribano, J. M. (2006). Optimization and validation of recombinant serological tests for African Swine Fever diagnosis based on detection of the p30 protein produced in Trichoplusia ni larvae. J Clin Microbiol 44(9), 3114-21.

Perez-Filgueira, D. M., Resino-Talavan, P., Cubillos, C., Angulo, I., Barderas, M. G., Barcena, J., and Escribano, J. M. (2007). Development of a low-cost, insect larvae-derived recombinant subunit vaccine against RHDV. Virology 364(2), 422-30.

Rose, R. C., Bonnez, W., Da Rin, C., McCance, D. J., and Reichman, R. C. (1994). Serological differentiation of human papillomavirus types 11, 16 and 18 using recombinant virus-like particles. J Gen Virol 75 ( Pt 9), 2445-9.

Rose, R. C., Bonnez, W., Reichman, R. C., and Garcea, R. L. (1993). Expression of human papillomavirus type $11 \mathrm{L1}$ protein in insect cells: in vivo and in vitro assembly of viruslike particles. J Virol 67(4), 1936-44.

Sigurdsson, K., Taddeo, F. J., Benediktsdottir, K. R., Olafsdottir, K., Sigvaldason, H., Oddsson, K., and Rafnar, T. (2007). HPV genotypes in CIN 2-3 lesions and cervical cancer: a population-based study. Int J Cancer 121(12), 2682-7.

Volpers, C., Schirmacher, P., Streeck, R. E., and Sapp, M. (1994). Assembly of the major and the minor capsid protein of human papillomavirus type 33 into virus-like particles and tubular structures in insect cells. Virology 200(2), 504-12.

White, W. I., Wilson, S. D., Bonnez, W., Rose, R. C., Koenig, S., and Suzich, J. A. (1998). In vitro infection and type-restricted antibody-mediated neutralization of authentic human papillomavirus type 16. J Virol 72(2), 959-64.

White, W. I., Wilson, S. D., Palmer-Hill, F. J., Woods, R. M., Ghim, S. J., Hewitt, L. A., Goldman, D. M., Burke, S. J., Jenson, A. B., Koenig, S., and Suzich, J. A. (1999). Characterization of a major neutralizing epitope on human papillomavirus type 16 L1. J Virol 73(6), 4882-9.

Xi, S. Z., and Banks, L. M. (1991). Baculovirus expression of the human papillomavirus type 16 capsid proteins: detection of L1-L2 protein complexes. J Gen Virol 72 ( Pt 12), 2981-8.

Zhou, J., Stenzel, D. J., Sun, X. Y., and Frazer, I. H. (1993). Synthesis and assembly of infectious bovine papillomavirus particles in vitro. J Gen Virol 74 ( Pt 4), 763-8. 


\section{Figures}

(a) $\mathrm{BacNi} B a c L 1 \mathrm{H}$ (b)
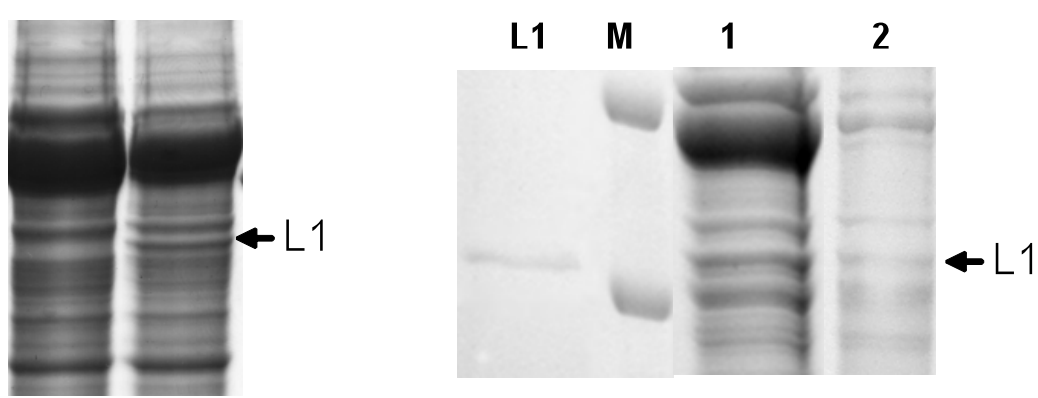

(c)

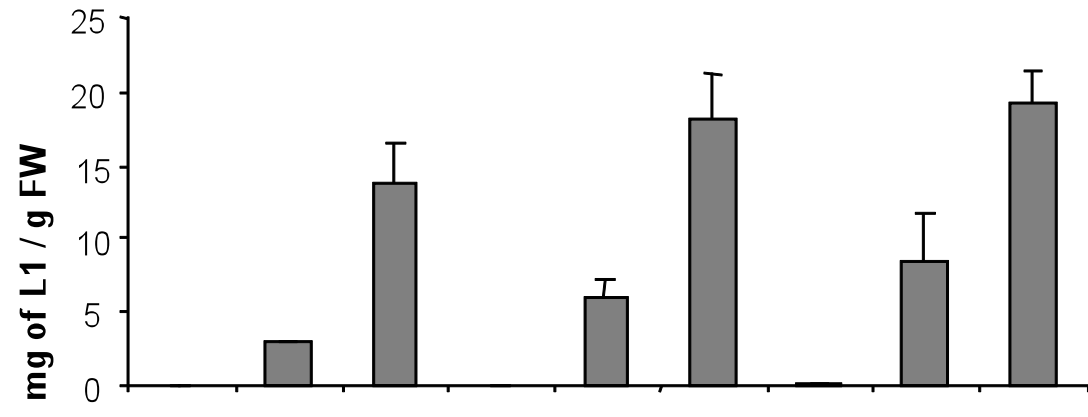

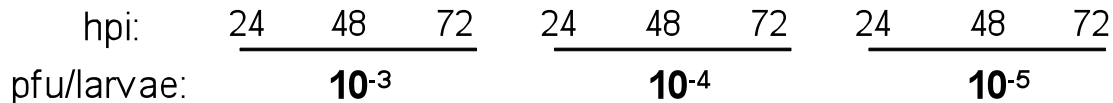

(d)

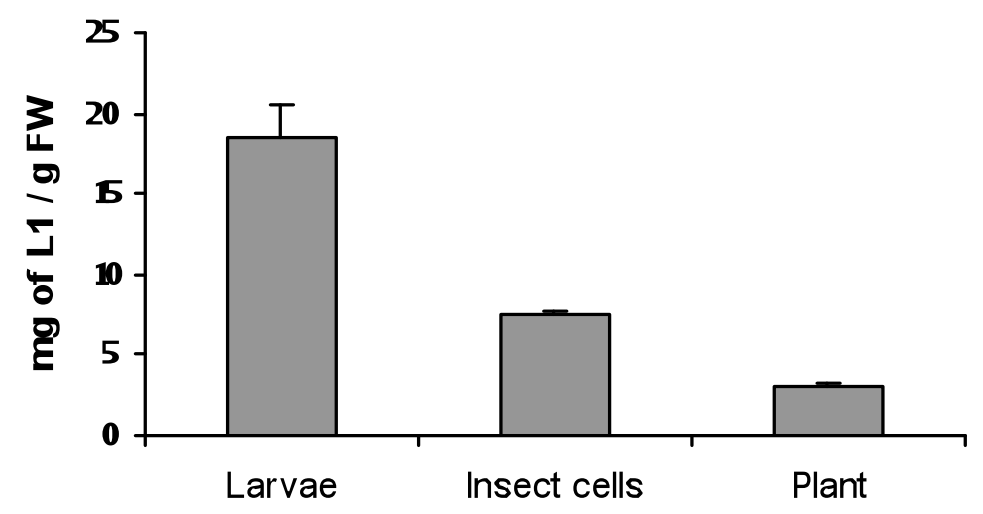

Figure 1 
(a)

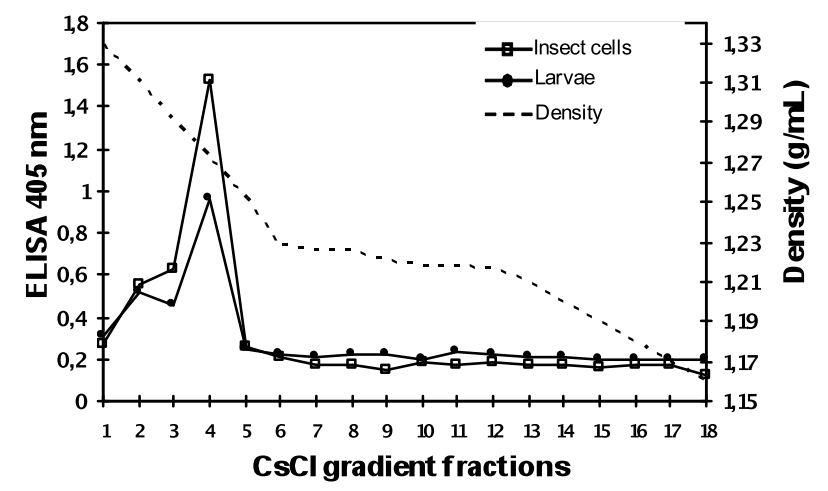

(b)

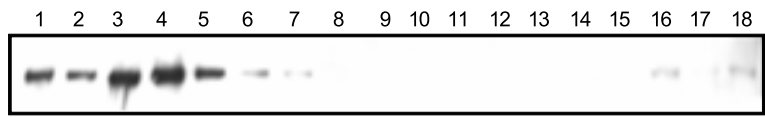

(c)
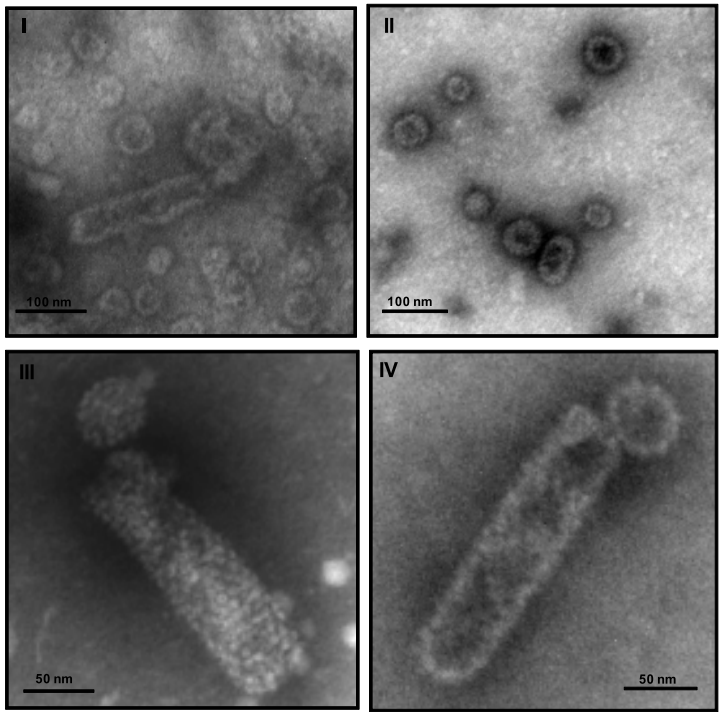

(d)

Sucrose gradient fractions

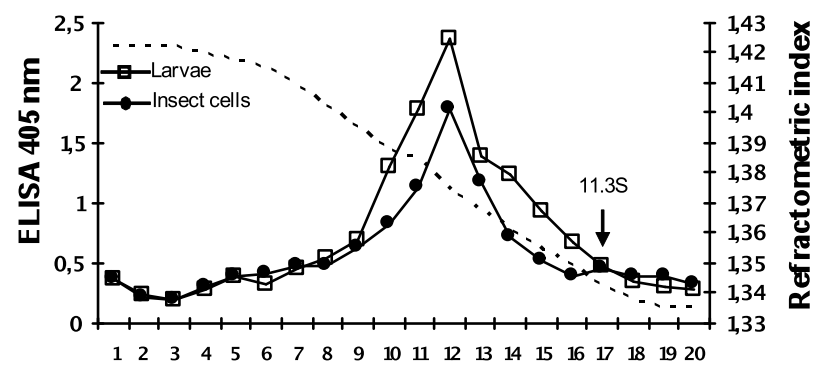

Figure 2 


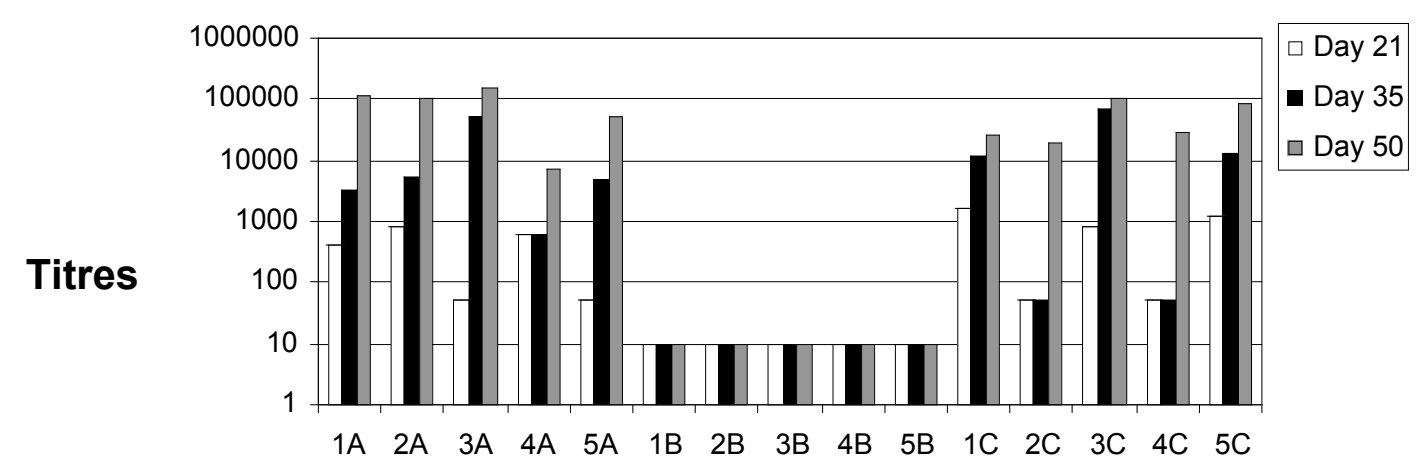

Mouse-Group

Figure 3 


\section{Legend to}

\section{figures}

Figure 1. L1 protein expression. (a) Coomassie and Immunoblot analysis of L1 protein expression in infected larvae. BacNi is used as negative control of the infection. (b) Coomassie of soluble (1) and insoluble (2) proteins. Proteins were extracted from $0.5 \mathrm{mg}$ larvae fresh weight/well were load. Purified 6xHis-L1 expressed in insect cells (L1) was used as a positive control $(2 \mu \mathrm{g})$. (c) Timedose assays measured by ELISA and immunoblot. (d) L1 accumulation in larvae analysed by ELISA. The result is the average of the analysis of three independent larvae batches infected with baculovirus. As comparison, expression in chloroplast transgenic tobacco plants and baculovirus-infected insect cells is also shown. In insect cells $1 \mathrm{~g}$ of pelleted cells comes from $55 \mathrm{ml}$ of culture.

Figure 2. Purification of VLPs from Trichoplusia larvae. (a) Detection of L1 by ELISA in fractions of a $\mathrm{CsCl}$ gradient (fraction 1 corresponds to the bottom tube). The Cam Vir-1 anti-L1 mAb was used as primary antibody. The dotted line indicates the density of the different fractions. (b) Immunoblot of the different fractions of larvae extracts using the same antibody. The band corresponds to the L1 monomeric form. (c) Electron microscopy of negatively stained VLPs from fraction 4 of the $\mathrm{CsCl}$ gradient. I and III from larvae extracts; II and IV from insect cell extracts. (d) Sucrose sedimentation analysis of L1 derived from larvae extracts, detected by ELISA with the Cam Vir-1 mAb. Soluble proteins from larvae and insect cells were fractionated by sucrose gradient centrifugation (fraction 1 corresponds to the tube bottom). The dotted line indicates the refractive index of the different fractions. The arrow indicates the fraction in which the standard (corresponding to capsomers' size) migrated. 
Figure 3. Analysis of the L1-induced antibody response in mice. Titres of antibodies induced by larvae-derived L1 protein against native yeast-derived VLPs. Balb/c mice were intraperitoneally immunized. Groups of mice immunized with A: partially purified VLPs from larvae; $\mathbf{B}$ partially purified negative control-larvae extracts; C: partially purified VLPs from insect cells. Data are presented as measures for individual mouse and standard errors $(n=2)$ are the result of two repetitions. 